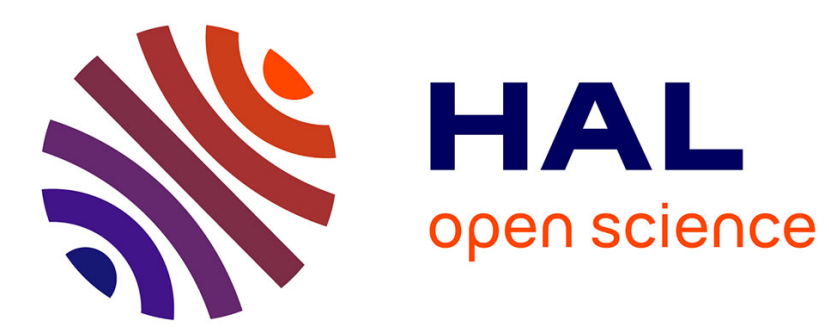

\title{
Generation of polychromatic radially and azimuthally polarized beams
}

T. Grosjean, M.A. Suarez, A. Sabac

\section{To cite this version:}

T. Grosjean, M.A. Suarez, A. Sabac. Generation of polychromatic radially and azimuthally polarized beams. Applied Physics Letters, 2008, 93 (23), pp.231106. 10.1063/1.3040056 . hal-00455153

\section{HAL Id: hal-00455153 \\ https://hal.science/hal-00455153}

Submitted on 6 May 2021

HAL is a multi-disciplinary open access archive for the deposit and dissemination of scientific research documents, whether they are published or not. The documents may come from teaching and research institutions in France or abroad, or from public or private research centers.
L'archive ouverte pluridisciplinaire HAL, est destinée au dépôt et à la diffusion de documents scientifiques de niveau recherche, publiés ou non, émanant des établissements d'enseignement et de recherche français ou étrangers, des laboratoires publics ou privés. 


\title{
Generation of polychromatic radially and azimuthally polarized beams
}

\author{
T. Grosjean, ${ }^{\text {a) }}$ M. Suarez, and A. Sabac ${ }^{\text {b) }}$ \\ Département d'Optique P.M. Duffieux, Université de Franche-Comté, Institut FEMTO-ST, \\ UMR 6174 CNRS, 16 route de Gray, 25030 Besançon cedex, France
}

(Received 13 October 2008; accepted 11 November 2008; published online 9 December 2008)

\begin{abstract}
We propose a fiber-based system aimed at generating radially and azimuthally polarized beams over spectral ranges of several tens of nanometers. The device is either adaptable to polychromatic sources such as supercontinuum fiber lasers or to tunable ones (optical parametric oscillator, laser diode, etc.). A preliminary version of the system has been engineered to work in the visible portion of the spectrum. The concept proposed here can be adapted to the infrared or blue-UV spectral domains. As a starting application, polychromatic Bessel beams of the first order have been produced. (C) 2008 American Institute of Physics. [DOI: 10.1063/1.3040056]
\end{abstract}

For 20 years, considerable improvements have been brought to the generation of radially polarized beams. The astonishing development of radial polarizers has been motivated by some pioneering studies which have proven a high added value of radially polarized beams in various fields of research. ${ }^{1-5}$ Nowadays, a large panel of systems are available for producing radially polarized beams at the visible, ${ }^{6-8}$ infrared, ${ }^{9-11}$ and terahertz frequencies. ${ }^{12}$ Most of these works are devoted to the generation of monochromatic beams. Polychromatic radially and azimuthally polarized beams may offer other perspectives in far-field and near-field microscopies, nano-optics, plasmonics, and spectroscopy. Unfortunately, only a very limited number of devices allow the generation of those kinds of light beams. The main system is based on the transmission of a polychromatic beam through a double conical reflector system. ${ }^{13}$ However, this original device radiates a discontinuous ring shape beam, which seems to be difficult to be applied without a beam reshaping with a specially designed optics.

In this paper, we propose a linear-to-radial polarization converter adaptable to polychromatic sources such as tunable or supercontinuum lasers. The system radiates a polychromatic Laguerre-Gauss beam ready to use for optical applications. The preliminary version of the system exhibits a bandwidth of about $150 \mathrm{~nm}$ in the visible portion of the spectrum. The device can be transposed to other spectral ranges such as the near-infrared or blue-UV domains. By projecting those beams onto an axicon, we obtained polychromatic Bessel beams of the first order, which find interesting applications in microscopy, spectroscopy, and particle trapping and guiding.

The principle of the proposed system is based on the ability of step index optical fibers to generate radially and azimuthally polarized beams ${ }^{14-16}$ and to transmit supercontinuum of light. ${ }^{17}$ When its core diameter is properly chosen, an optical fiber can propagate four modes among which we find the annular modes $\mathrm{TM}_{01}$ radially polarized, $\mathrm{TE}_{01}$ azimuthally polarized, and $\mathrm{HE}_{21}$ with hybrid polarization. The most direct way for producing a radially or azimuthally polarized beam consists of exciting $\mathrm{TM}_{01}$ or $\mathrm{TE}_{01}$, respectively.

\footnotetext{
${ }^{a)}$ Electronic mail: thierry.grosjean@univ-fcomte.fr.

${ }^{b}$ Present address: Institut des Nanotechnologies de Lyon, INSA de Lyon, UMR 5270 CNRS-ECL-INSA-UCBL, 7 avenue Jean Capelle, 69621 Villeurbanne Cedex, France.
}

However, it seems that the selection of the third annular mode $\mathrm{HE}_{21}$ is easier to achieve. ${ }^{16}$ Since the hybrid to radial or azimuthal polarization conversion can be obviously realized with a half-wave retardation plate properly oriented, this indirect solution should be more adapted for fulfilling our objectives.

The spectral bandwidth $\Delta \lambda$ of the step index optical fiber used as an achromatic axial polarizer is given by ${ }^{18}$

$$
\begin{aligned}
\Delta \lambda & =\lambda_{1}-\lambda_{2} \\
& =2 \pi a \sqrt{n_{1}^{2}-n_{2}^{2}}\left(\frac{1}{2.41}-\frac{1}{3.83}\right),
\end{aligned}
$$

where $a$ is the core diameter and $n_{1}$ and $n_{2}$ are the core and cladding indices. $\lambda_{1}$ and $\lambda_{2}$ are the cutoff wavelengths of the mode of interest $\mathrm{HE}_{21}$ and the next one $\mathrm{EH}_{11}$ in the fiber mode classification, respectively. Beyond $\lambda_{2}$, the fiber propagates higher modes and the mode selection becomes much more difficult. As an example, for optical fibers designed to be monomode at 820 and $780 \mathrm{~nm}$, the spectral bandwidths are typically limited to the ranges 470-750 and $450-715 \mathrm{~nm}$, respectively. Most of the visible portion of the spectrum and a little part of the infrared one are covered by the spectral bandwidth of these fibers. Therefore, the generation of quasi-white-light radially or azimuthally polarized can be expected. Note that $\Delta \lambda$ represents the largest bandpass of the linear-to-axial polarizers that use optical fibers.

The fiber-based system shown here is derived from the one proposed in Ref. 16 for generating monochromatic axially polarized beams. The purpose here is to extend to the largest spectral bandwidth the wavelength-dependent selection process of $\mathrm{HE}_{21}$ that has been previously suggested. The larger spectral bandpass is limited by the optical fiber itself. The preliminary version of the resulting device is engineered to work at visible frequencies (see Fig. 1). The source is a supercontinuum of light obtained by coupling a high power pulsed laser beam into a specific optical fiber aimed at generating a polychromatic light source over several tens of nanometers. Tunable laser sources can also be used. First, the depolarized collimated beam passes through a linear polarizer before crossing a discontinuous phase element (DPE). The DPE consists of the association of two achromatic halfwave retardation plates set in contact to each other so that the fast axis of one plate is oriented parallel to the slow axis of the second one. In our case, the DPE is achromatic over the 


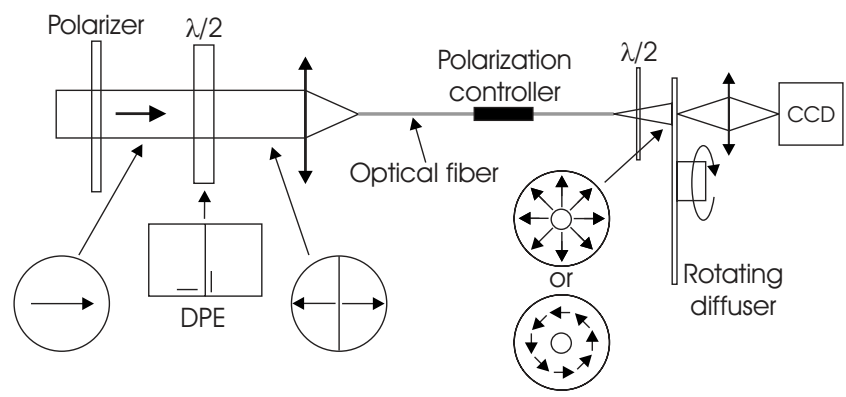

FIG. 1. Scheme of the experimental setup of generation and imaging of the polychromatic radially polarized beam.

entire portion of the visible spectrum $(400-700 \mathrm{~nm})$. This combination leads to a $\pi$-phase retardation between the two halves of a white light beam, which passes through the DPE at normal incidence with a polarization direction along one of the two axes of the crystal pieces. Second, the modified beam is coupled into a conventional step index fiber (Spectran, monomode at $820 \mathrm{~nm}$ ) with an apochromatic objective (Nikon, $\times 4,0.16$ ) for limiting chromatic dispersion effects in the focusing process. Due to the fiber optical properties, the phase difference induced by the DPE leads to the cancellation of the fundamental mode and the transmission of the three annular modes $\mathrm{TM}_{01}, \mathrm{TE}_{01}$, and $\mathrm{HE}_{21}$ for the quasioverall-visible spectrum. Finally, the energy conversion from $\mathrm{TM}_{01}$ and $\mathrm{TE}_{01}$ to $\mathrm{HE}_{21}$ is carried out by creating the right birefringence into the fiber with a fiber polarization controller aimed at twisting and compressing the optical fiber at will (Newport, model F-POL-IL). Note that this latter empirical process is wavelength dependent and will be the main limit of the spectral bandwidth of the proposed system. The hybrid to radial or azimuthal polarization conversion is obtained with the same half-wave retardation plate as the ones used in the DPE. The acquisition is realized with a colored charged coupled device (CCD) camera. In order to avoid speckle in the images, the output beam is projected onto a rotating glass diffuser.

The adjustment process of the system is divided into four steps. First, the polychromatic light is injected into the fiber when the DPE is translated laterally so that the input beam does not cross the phase step. In this configuration, the energy is coupled to the fundamental mode. In a second time, the DPE is translated so that the $\pi$-phase step is placed into the beam cross section. This adjustment is stopped when the output beam shows the lower intensity at its center. Then, the $\mathrm{HE}_{21}$ mode is selected by means of the fiber polarizer. This third step is controlled by projecting the beam onto a rotating analyzer coupled to a screen. Finally, the half-wave plate is placed at the system output in order to obtain a radially or azimuthally polarized doughnut beam.

The so-obtained polychromatic radially polarized beam is shown in Fig. 2. Figure 2(a) displays the free space doughnut beam and Fig. 2(b) shows the chromatic spectrum. The presence of a clearly marked central dark spot proves that the fundamental mode is canceled over a large part of the visible spectrum. The polarization of the polychromatic doughnut is characterized in Figs. 2(c)-2(f) by inserting an analyzer (linear polarizer) before the diffuser and imaging the beam for four different analyzer directions. These directions are indicated by white arrows in the images. The high visibility of the two-grain structures, which follow the analyzer axis,
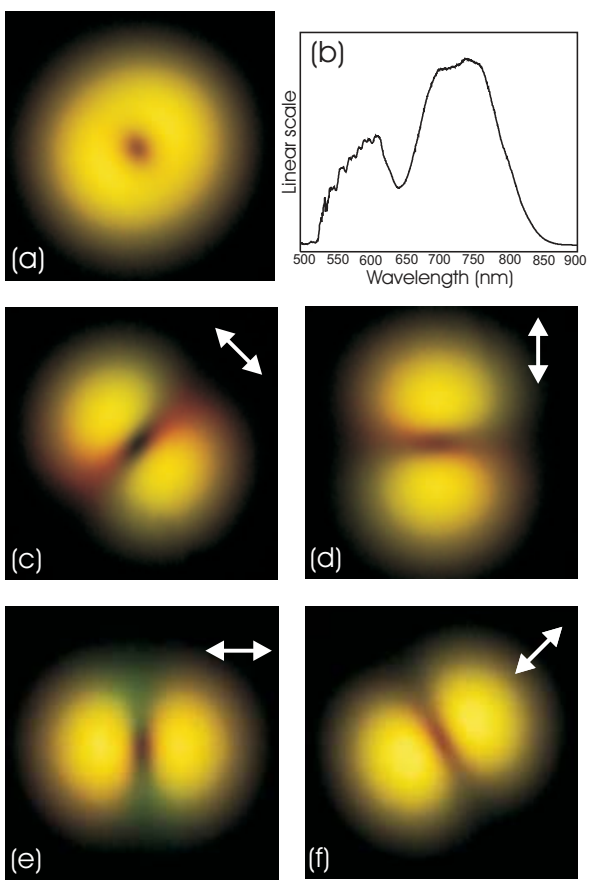

FIG. 2. (Color) (a) Image of the radially polarized polychromatic beam. (b) Chromatic spectrum of the optical source (linear scale). [(c)-(f)] Image of the radially polarized beam after passing through an analyzer whose axis directions are indicated by white arrows.

proves that the output polychromatic beam is radially polarized over a large part of the visible frequencies. The measured spectral bandwidth of the system, about $150 \mathrm{~nm}$, is twofold smaller than the one of the fiber used as an axial polarizer. This limitation is due to the dispersive effect of the fiber polarization controller.

Among the possible applications for this kind of doughnut beams, a direct one is the generation of polychromatic Bessel beams of the first order. Zeroth-order polychromatic Bessel beams have been recently studied and produced for applications in imaging, micromanipulation, and interferometry. ${ }^{19}$ By using a compensated spatial light modulator, it has been shown that polychromatic Bessel beams of larger orders can be created through the generation of optical vortices. $^{20}$ The use of polychromatic radially polarized beams leads to first-order Bessel beams no more linearly (or circularly) polarized, which do not carry any angular momentum. Such properties are, for example, of high interest for the optical characterization of nanoantennas. ${ }^{21}$ In the frame of this study, the Bessel beam is obtained by transmitting the polychromatic radially polarized beam through an axicon of low numerical aperture. ${ }^{22}$ Figure 3 (a) displays the polychromatic Bessel beam of the first order and Figs. 3(b)-3(d) show the records when the beam passes through interferential filters centered at (b) 550, (c) 600, and (d) $650 \mathrm{~nm}$ and of $40 \mathrm{~nm}$ bandpass. We see that the Bessel beam exhibits a bright doughnut with a central dark hole over a spectral extent of about $150 \mathrm{~nm}$, which is consistent with the spectral bandwidth of the axial polarizer.

This paper presents a passive fiber-based system able to convert a polychromatic linearly polarized beam into a polychromatic radially or azimuthally polarized one. This device can be used with various polychromatic sources such as tunable or supercontinuum lasers. The principle of this axial polarizer has been demonstrated at visible frequencies but it 

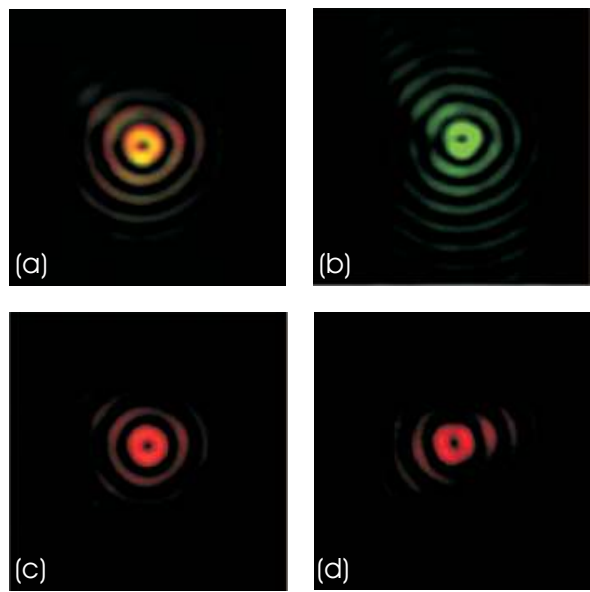

FIG. 3. (Color) (a) Image of the polychromatic Bessel beam of the firstorder. [(b)-(d)] Images at 550, 600, and $650 \mathrm{~nm}$, respectively. The bandpasses of the filters used for (b)-(d) are $40 \mathrm{~nm}$ large.

may be adapted to the near-infrared or blue-UV domains. Up to now, the maximum spectral bandwidth of the device, which was built only with commercial elements, has been measured to values around $150 \mathrm{~nm}$ in the visible spectral range. Starting from these radially polarized beams, the generation of polychromatic Bessel beams of the first order has been demonstrated. Their combined polarization, spectral, and nondiffracting properties are attractive for applications in imaging, nano-optics, and spectroscopy. They can also be used as the counterpart of the already produced polychromatic Bessel beams of the first order, which carry angular momentum, for comparative studies in micromanipulation. Conventional focusing or propagation of polychromatic radially and azimuthally beams finds numerous applications in plasmonics, nano-optics, near-field microscopy or spectroscopy, etc.

This work is supported by the Agence Nationale de la Recherche under Contract No. ANR-07-NANO-036.

${ }^{1}$ S. Quabis, R. Dorn, M. Eberler, O. Glöckl, and G. Leuchs, Opt. Commun. 179, 1 (2000)

${ }^{2}$ L. Novotny, M. R. Beversluis, K. S. Youngworth, and T. G. Brown, Phys. Rev. Lett. 86, 5251 (2001).

${ }^{3}$ V. G. Niziev and A. V. Nesterov, J. Phys. D: Appl. Phys. 32, 1455 (1999).

${ }^{4}$ Q. Zhan, Opt. Express 12, 3377 (2004).

${ }^{5}$ I. Moshe, S. Jackel, and A. Meir, Opt. Lett. 28, 807 (2003).

${ }^{6}$ M. Stalder and M. Schadt, Opt. Lett. 21, 1948 (1996).

${ }^{7}$ R. Oron, S. Blit, N. Davidson, A. A. Friesem, Z. Bomzon, and E. Hasman, Appl. Phys. Lett. 77, 3322 (2000).

${ }^{8}$ K. Yonezawa, Y. Kozawa, and S. Sato, Opt. Lett. 31, 2151 (2006).

${ }^{9}$ S. C. Tidwell, D. H. Ford, and W. D. Kimura, Appl. Opt. 29, 2234 (1990).

${ }^{10}$ A. V. Nesterov, V. G. Niziev, and V. P. Yakunin, J. Phys. D 32, 2871 (1999).

${ }^{11}$ T. Moser, H. Glur, V. Romano, M. A. Ahmed, F. Pigeon, O. Parriaux, and Th. Graf, Appl. Phys. B: Lasers Opt. 80, 707 (2005).

${ }^{12}$ G. Chang, C. J. Divin, C.-H. Liu, S. L. Williamson, A. Galvanauskas, and T. B. Norris, Opt. Lett. 32, 433 (2007).

${ }^{13}$ A. Shoham, R. Vander, and S. G. Lipson, Opt. Lett. 31, 3405 (2006).

${ }^{14}$ T. Grosjean, D. Courjon, and M. Spajer, Opt. Commun. 203, 1 (2002).

${ }^{15} \mathrm{G}$. Volpe and D. Petrov, Opt. Commun. 237, 89 (2004).

${ }^{16}$ T. Grosjean, A. Sabac, and D. Courjon, Opt. Commun. 252, 12 (2005).

${ }^{17}$ S. V. Chernikov, Y. Zhu, J. R. Taylor, and V. P. Gapontsev, Opt. Lett. 22, 298 (1997).

${ }^{18}$ E. Snitzer, J. Opt. Soc. Am. 51, 491 (1961).

${ }^{19}$ P. Fischer, C. Brown, J. Morris, C. López-Mariscal, E. Wright, W. Sibbett, and K. Dholakia, Opt. Express 13, 6657 (2005).

${ }^{20}$ J. Leach, G. M. Gibson, M. J. Padgett, E. Esposito, G. McConnell, A. J. Wright, and J. M. Girkin, Opt. Express 14, 5581 (2006).

${ }^{21}$ T. Grosjean, A. Fahys, M. Suarez, D. Charraut, and D. Courjon, J. Microsc. 229, 354 (2008).

${ }^{22}$ R. M. Herman and T. A. Wiggins, J. Opt. Soc. Am. A 8, 932 (1991). 